\title{
The processing of fused infrared and vision images of a welding arc for fault detection*
}

by M. Fidali, W. Jamrozik

\begin{abstract}
Silesian University of Technology ,Department of Fundamentals of Machinery Design, Konarskiego 18a,
\end{abstract} 44-100 Gliwice, Poland, \{ marek.fidali; wojciech.jamrozik\}@polsl.pl

\begin{abstract}
The article deals with the processing of fused infrared and vision images of a welding arc. The considered sequences of infrared and vision images were gathered during a series of laboratory experiments. Different welding instabilities were simulated during the experiments. The aim of the research was to diagnose the welding process based on the results of the processing of fused images. Three methods of analysis of fused images were considered in order to generate diagnostic signals. The first method deals with statistical estimation of pixel values of segmented welding arc area. In the second method topological parameters of the area of welding arc was estimated. The third method was based on the analysis of horizontal profile of welding arc area. In this case several geometrical features were proposed. Extracted features of fused image were estimated. It enabled to perform classification of welding state. Obtained results showed that proposed methods of analysis allowed generation of useful diagnostic signals which could be used for further fault detection and localization purposes.
\end{abstract}

\section{Introduction}

During the welding process broadband electromagnetic radiation including ultraviolet, visible light and the infrared is emitted. Wide electromagnetic radiation allows use of different imagining devices for the monitoring of welding process. Application of imagining devices permit observation and gathering images representing e.g.: the shape of the welding arc in visible light as well as near infrared; temperature distribution of plasma and of gases in the near infrared; the shape and temperature distribution of the welding pool and also welding seam and heat-affected zone in the far infrared range, as well as in the visible light.

Images acquired independently in different bands of the electromagnetic radiation are a source of specific and often mutually complementary information over realized process. Thus more and more often images are acquired simultaneously and merged together for the purpose of the creation of the new synthetic image.

Joining of the information two or more images is called image fusion. Image fusion permits to limit the uncertainty and the amount of redundant information in the output image, preserving the relevant information from input images. It makes the image fusion very useful tool for the use in machine vision systems which use automatic image processing and inference algorithms.

The image fusion is well known for many years and most often consists in the realization of two basic operations: images registration and images aggregation. There exist many different algorithms realizing the process of image fusion [19][13][20]. Some image fusion algorithms found application in the contemporary infrared cameras with the embedded visible light camera [4]. Such technology supports the camera operator in interpretation of the observed infrared images. Fusion of infrared and vision images found also application in military and civil surveillance systems for searching and tracking objects. In the welding technology, image fusion was applied in the joining of the X-ray and ultrasonic images of the welds [3]. Such operation made estimation of the welded joints quality more effective, allowing detection of internal defects. Fusion of infrared and vision images could be also used in on-line diagnostics of the welding process [2][9]. Previous research of the authors showed that the quality of the infrared and vision images after fusion depends on applied method of images registration and aggregation. In [8] [9] [10] evaluation and selection of optimal algorithms for registration and aggregation of the infrared and vision images allowing the obtainment of fused images representing welding arc that consist features useful for effective estimation of welding process in on-line mode.

The aim of research presented in the paper was the verification of proposed image processing methods of fused infrared and vision images of welding arc for the purpose of their application to detection and identification of welding process instabilities.

In the paper three ways of the analysis of fused infrared and vision images of welding arc were presented. The statistical and topological analysis of regions that need previous application of segmentation of fused image was utilized. Fused image profiles were also analysed in order to extract features useful for detection of welding faults.

An example of application of proposed methods to sequences of fused infrared and vision images was also presented in the article. Infrared and vision images utilised during the research were taken during several experiments with simulation of different welding faults caused by intentionally introduced instabilities of process parameters.

Comparison of the results of analysis of fused images with results of independent analysis of infrared and vision images was also performed in the article. 


\section{Acquisition and fusion of infrared and vision images of welding arc}

In order to fuse and analyse infrared and vision images of welding arc it was necessary to gather the images and select the most effective fusion algorithm. Infrared and vision images were taken during welding of plates made of

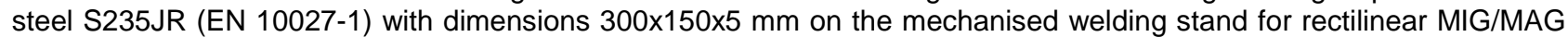
welding equipped in microprocessor controlled welding machine Castolin TotalArc 5000. The edges of the joined plates were bevelled at an angle of $\alpha=60^{\circ}$ and the offset between them was $b=1,0 \mathrm{~mm}$.

For welding purposes a solid electrode wire with a diameter of $0,2 \mathrm{~mm}$ (Castolin CastoMag 45255) and a shield gas M21 (82\%Ar+18\% $\mathrm{CO}_{2}$ ) were used. Nominal welding parameters are presented in Tab. 1.

Table 1. Optimal MAG welding parameters

\begin{tabular}{|c|c|c|c|c|c|}
\hline $\begin{array}{c}\text { Welding current } \\
{[\mathrm{A}]}\end{array}$ & $\begin{array}{c}\text { Welding voltage } \\
{[\mathrm{V}]}\end{array}$ & $\begin{array}{c}\text { Welding speed } \\
{[\mathrm{cm} / \mathrm{min}]}\end{array}$ & $\begin{array}{c}\text { Wire feeding rate } \\
{[\mathrm{m} / \mathrm{min}]}\end{array}$ & $\begin{array}{c}\text { Shield gas flow } \\
{[\mathrm{l} / \mathrm{min}]}\end{array}$ & $\begin{array}{c}\text { Electrode outlet } \\
{[\mathrm{mm}]}\end{array}$ \\
\hline 240 & 25 & 32 & 7,4 & 15 & 15 \\
\hline
\end{tabular}

During the welding, infrared and vision images were synchronically acquired with rate $50 \mathrm{fps}$ by use of uncooled infrared camera Infratec VarioCam Head with resolution $640 \times 480 p x$ equipped in lens $f=50 \mathrm{~mm}$ and visible light CCD camera ImagingSource DMK21AF04 with resolution $640 \times 480 p x$ and lens with focal length $f=25 \mathrm{~mm}$. Camera's lenses were equipped in a suitable filters suppressing the excess of the electromagnetic radiation and protecting it against splatters generated during welding. Cameras connected to the computers and images acquisition process was controlled by software developed in LabView environment.

Series of experiments simulating different faults of welding process were carried out. It permitted to record the collection of sequences of infrared and vision images for 12 different states of welding process classified in the following way:

S1 - Correct welding process.

S2 - Welding with decay of the shielding gas flow.

S3 - Welding of the plates with distinct outbreaks of atmospheric corrosion on the welded surfaces.

S4 - Welding with use of corroded wire.

S5 - Welding of plates with irregularities of the plate edges from side of the weld root.

S6 - Welding of plates with oil contamination.

S7 - Welding with deviation of current.

S8 - Welding of plates with different offset intervals.

S9 - Welding with deviation of voltage.

S10 - Welding of the plates with improper welding groove geometry.

S11 - Welding with deviation of speed.

S12 - Welding with use of worn rollers of wire feeder.

Acquired infrared and vision images were fused. The fusion operation demanded image registration and next aggregation with use of methods selected during earlier authors' research [8][9].

Image registration was performed by use of edge orientation map algorithm based on objective entropy function and 3D histogram build for the edge orientation maps and pixel intensities values [11]. Objective function represented the uncertainty about the edge orientation coincidence of the corresponding intensity pair as well as the uncertainty between the intensity values of two images. This method can be used for indexed as well as gray level images.

The aggregation of the information contained in registered input images were performed by use of algorithm based on shift invariant modified wavelet transforms $(\mathrm{SIH})$ [18]. In this algorithm input images are decomposed into a shift invariant wavelet structures using the wavelet frames concept with the Haar wavelet. Consecutive images are not decimated, which causes that its content is highly redundant. Fused images are created by application of Brut rule [1]. It allows to join images by application of pixel averaging and selection according to change of neighbouring pixels variance.

In figure 1, exemplary infrared, vision and fused images of welding arc were presented. Presented images were gathered for different welding process states. One can observe the differences in image content which strongly depends on welding process parameters and welding materials. 


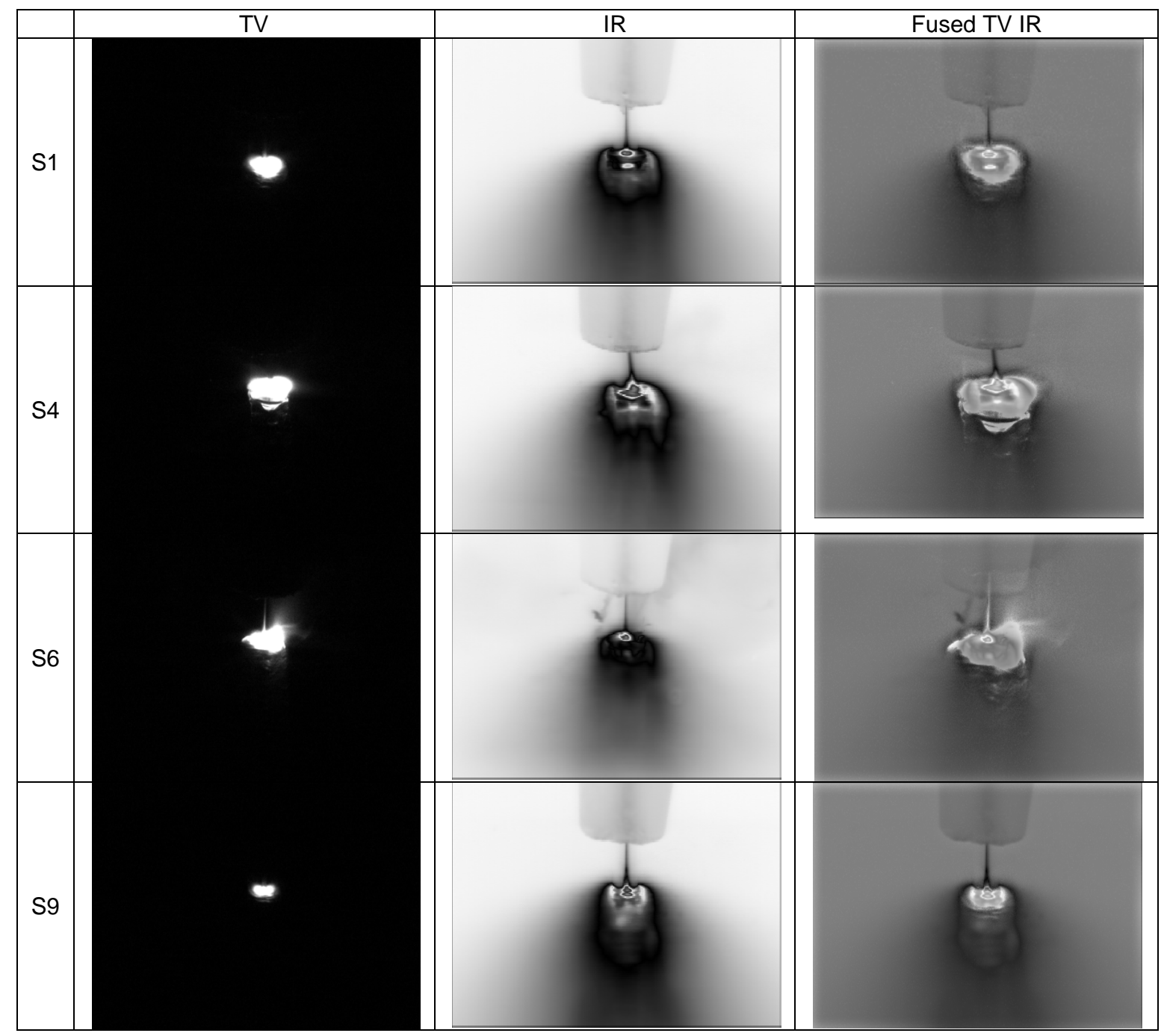

Fig. 1. Exemplary vision and infrared images of welding arc and result of their fusion recorded during different states of welding process

\section{The processing of the fused images of welding arc}

Diagnostic of the welding process based on fused infrared and vision images requires elaboration and/or selection of image processing and analysis methods allowing extraction of relevant diagnostic features from the images. There exist several examples of the analysis of fused images [12], however authors didn't found examples of analysis fused infrared and vision images of the welding arc for diagnostics of welding process purposes. The lack of methods of the analysis of fused images of welding arc should not be a limitation for the usage of existing infrared and vision image analysis methods [5][15] for these needs. There are several examples of the analysis of vision and infrared images of welding arc. The most often applied methods bases on the pixels profile along lines located on different regions of the image. For example in [21] there was described the application of horizontal line profile and isothermal maps for the detection of defects such as the lack of penetration and estimation of depth of penetration during Tungsten Inert Gas (TIG) welding. The authors of [17] proposed a method based on CCD thermography and specific image processing approach using line subpixel detection in order to control the HF soldering process. In [14] image processing algorithm for automatic identification and quantification of incomplete Penetration in Tungsten Inert Gas (TIG) welding was proposed. Developed image processing algorithm utilizes colour to gray level conversion, edge detection by Sobel filter mask and morphological processing such as dilation, erosion and region filling to detect and quantify the defect. For quantifying the defect such features as area, major axis length and minor axis length were used.

Existing image processing and analysis methods create very wide possibilities of analysis of welding arc fused images. A closer look at fused infrared and vision images presented in fig. 1 and fig. 2, leads to a conclusion that there are two kinds of information types: the first is connected with the distribution of light; the second is temperature distribution on a surface of a welding seem and the tip of a wire. This information is changing along observation time and is different for stable and unstable welding processes which can be seen in fig. 1 and fig. 2 where fused images of a 
welding arc in different process states are shown. Visible are noticeable differences in the shape and the size of the area of arc light and temperature distribution within welding pool. It permits one to conclude, that suitable analysis of the sequence of fused images of welding arc could make possible the identification of welding process instabilities.

a)

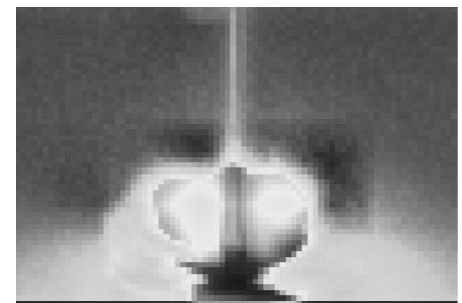

b)

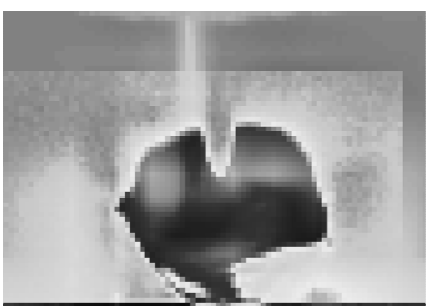

c)

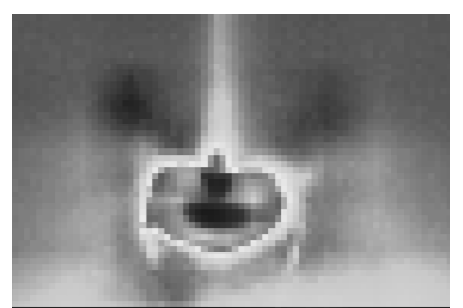

Fig.2. Examples of fused infrared and vision images taken during different instabilities of welding process: a) temporary problem in flow of shielding gas, b) rusted wire, c) temporary descent of welding current value

\subsection{Processing of the images before and after fusion}

The image fusion and then their further analysis demands consideration of several fundamental and important image processing operations. Processing operation could be performed both for input images before fusion as well as for picture being effect of the fusion.

Input images for fusion operation besides lack geometrical adjustment (relative translation and rotation) could have different representation and recording formats (color, gray level images); different sizes and resolutions and could be represented in different ranges of pixels values. Such variety of image parameters demands their unification before fusion process. The image fusion operation is usually carried out on array of numbers therefore the first step of image processing should be adjustment of sizes and resolution of images. Resolution adjustment could be performed by increasing resolution of the image with originally lower resolution with superresolution methods [7] or by decreasing resolution of one image with higher resolution than the second one. In fig. 3 the effect of the fusion (c) of infrared image (a) which resolution was lower than vision image (b) and situation were fused image (e) was created from infrared image (d) with increased resolution and vision image (b).

a)

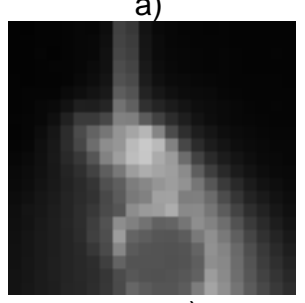

c)

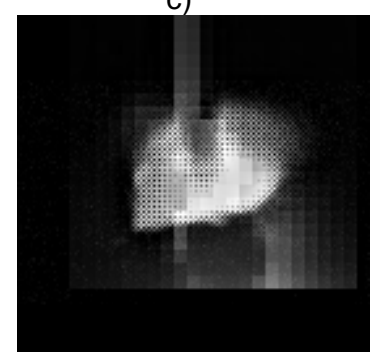

b)

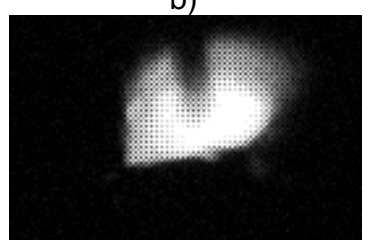

d)

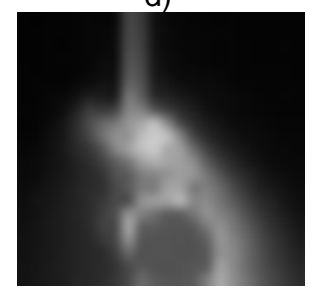

e)

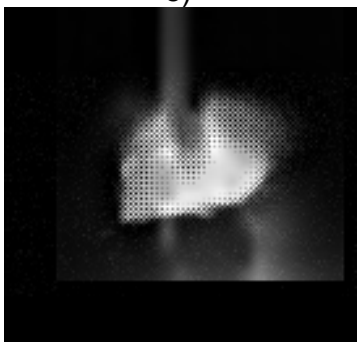

Fig.3. Example of influence of different input image resolution on fusion effect

In vision image (fig. 3b) one can see artefacts caused by existence of grid of colour filters placed on the detector surface of CCD colour camera which was used for acquisition of the image. In processing stage such undesirable noise can be removed by use of properly fitted filters (eg. Median filter).

Another important processing operation performed on images before fusion is selection of a region of interest (ROI). Very often camera's field of view covers wider area than is needed to analyze. Taking into account whole image areas for image fusion purpose is unfounded and could result undesirable quality of fused image. From this reason, it is necessary to select on the input images areas of interests before image fusion operation. Pixels into selected areas will be subject of further fusion and analysis.

At the selection of the region of interest one ought to take into account the translation between images which can occur during the image registration. The choice of the too small region of interest could cause that the mutual dislocation of images resultant during the registration could produce difficult to the elimination edges which can have the 
influence on results of the image aggregation and then results of the further analysis. The example of incorrectly selected regions of interest for images before fusion is presented in Fig. 2b. One can see the ages of input images.

Infrared images are very often represented as arrays of temperature values whereas monochromatic vision images are arrays of grey level values. Differences in ranges of pixel values of the images can lead to undesirable results of image fusion. From this reason it is purposeful to unify ranges of pictures pixel values before fusion. Such operation can be made by applying image normalisation.

In many cases is necessary to select the region of interest again after performed image fusion. This results from presence of image edges which could appear after translation of the images during their registration. Presence of such artefacts can influence further results of image processing and analysis. In Fig.4 is shown an example where fused images were processed using histogram equalisation. In the first case selection of region of interest was not performed. In the second case selection of ROI significantly influenced the histogram equalisation results.

Fused images

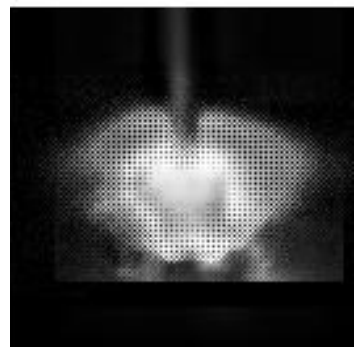

Fused images after processing

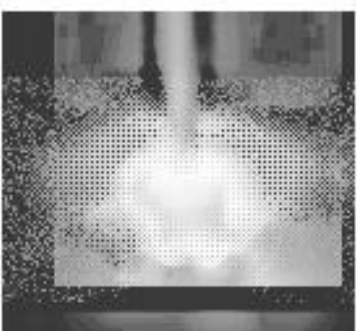

Selected ROI of fused images

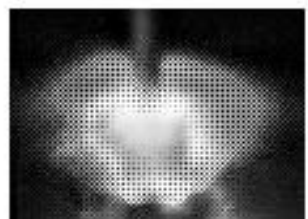

selected ROI of fused images after processing

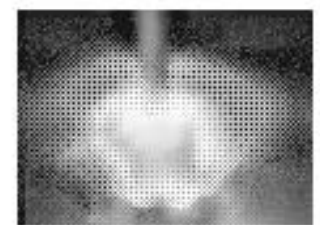

Fig.4. Example of influence of application of ROI selection on processing result of fused images

Processing operations like the region of interest selection, resizing or resampling are not all that can be applied to the images before and after the fusion. There are much more image processing operations like image equalization, filtering, thresholding, segmentation etc [5][15].

After image processing fused images are analysed in order to extract features necessary for detection and identification of welding faults.

\subsection{Statistical analysis of the arc area}

Fused images of welding arc carry the information about shape and size of area of arc light as well as temperature distribution of the welding pool and heat-affected zone. Such image structure allows its segmentation and selection of area of arc light and then the extraction e.g. statistical features, on the basis of pixel values in selected area. This approach permits to characterize e.g. the variability of temperatures in the area of the welding pool and tip of the electrode wire. The area can be defined once for reference image and applied for all remaining images in the sequence. The area can be also defined dynamically for each image in the sequence however it demands the formulation of correct segmentation criteria. The dynamic approach causes that the parameter describing the content of the area does not depend only from the value of pixels inside the area but also from sizes of this area. In fig. 4 the example of fused image with the marked area determined by use of segmentation was presented and in fig. 5 selected statistical features determined for fused images represented different faults of welding process are shown. The features values varied according to welding state changes.

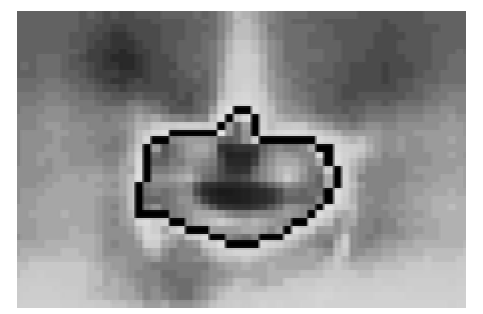

Fig. 5. Segmented area of arc light

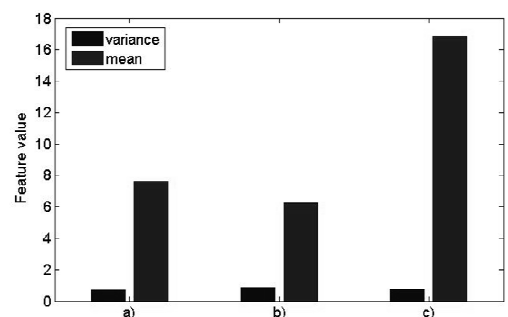

Fig. 6. Statistical features of segmented areas of arc

During the research, authors applied segmentation method based on the Otsu thresholding method. It permitted to define reference area for all considered images of acquired sequences. Within given area first and second order statistical features [6] [16] were determined. Considered 16 statistical features of first order like: mean and rms value, kurtosis, skewness, crest factor, ect. Second order statistic calculated on the basis of the co-occurrence matrix and this were the contrast, correlation, energy, entropy, homogeneity (inverse difference moment), variance, covariance. 


\subsection{Topological analysis of the arc area}

On fused images of welding arc, several characteristic areas like welding pool and arc light are visible. One observed that these areas changed their shapes during the welding. It allows to assume that features of shapes of the characteristic areas can be treated as a source of information about the welding process stability. For the purpose of extraction of this information it is necessary to:

- define one or several characteristic areas,

- extraction of areas with use of image segmentation,

- selection of parameters set suitable for extracted area estimation,

- calculation of feature values.

Proposed algorithm seems to be simple for a single image, however in the case of image sequence, where segmentation is required for each image, the task becomes more complex and needs special attention during selection of segmentation method. Authors applied previously mentioned segmentation based on Otsu thresholding.

To describe shapes of extracted areas commonly known topological parameters could be applied. Authors considered following features: major and minor diagonal, area, perimeter, shape factor, Ferret's factor, Malinowska's factor etc [5][15].

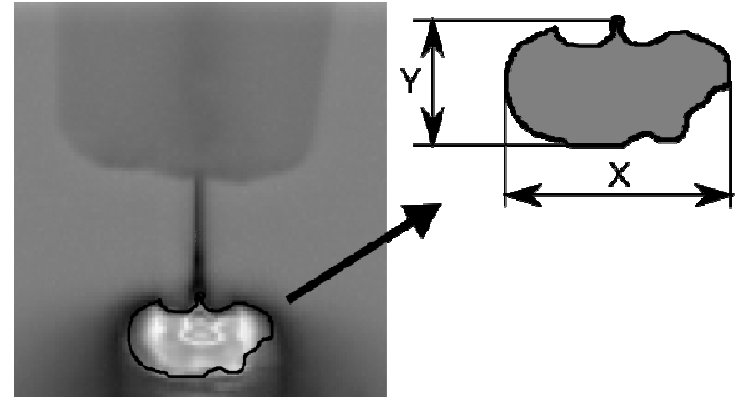

Fig. 7. Example of topological features of segmented areas of arc

\subsection{Analysis based on profile of the arc area}

In the case of fused images of welding arc a valuable source of information are pixel values distributions along line called profile. Profiles can be analysed in different ways. One of proposed approach concerns analyses of the horizontal profile of arc area. As shown in fig. 8 horizontal profile which crosses arc area and welding seem has a pseudo symmetrical character. It follows from properties of welding process. The disturbance in the symmetry of the profile can testify the appearance of the instability of the welding process. This assumption allowed defining the profile features which are the widths between abscissas of the characteristic points. Characteristic points correspond to the extremes of horizontal profile passing by image pixels with the maximum value. During the research following five features were considered:

- left and right external width (LEW, REW)

- left and right internal width (LIW, RIW)

- $\quad$ central width $(\mathrm{CW})$

Additionally one can also consider following features:

- left and right half width (LHW, RHW)

- $\quad$ total width (TW)
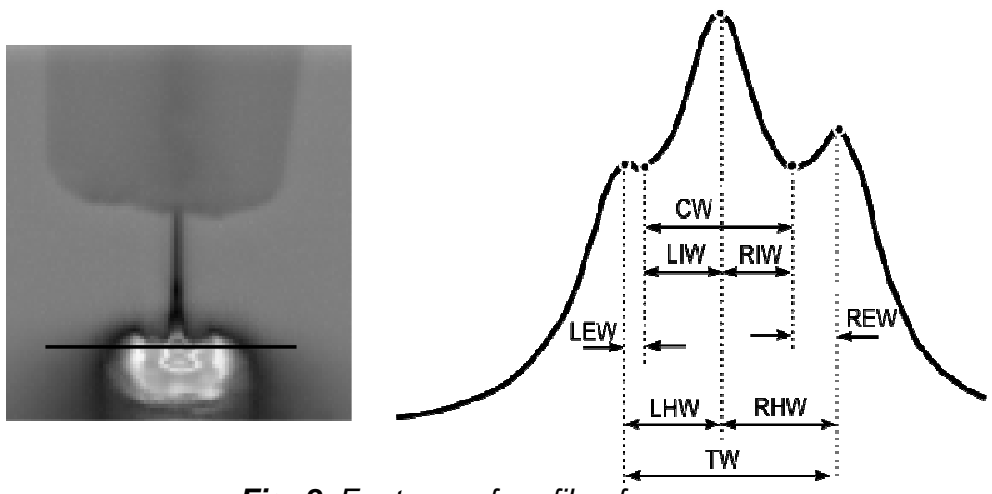

Fig. 8. Features of profile of arc area

The horizontal profile can be also useful for description of object geometry visible in the image and finding its characteristic points necessary for determination of different arc area features. In the case of author's research, the cumulative horizontal profile was applied to determine the position of the axis of symmetry of electrode wire (Fig. 9b). The position of wire axis of symmetry was necessary to determine the relative feature (MWD - max-wire distance) describing horizontal distance between position of the pixel with maximal value and wire axis of symmetry (Fig. 9a). 
a)

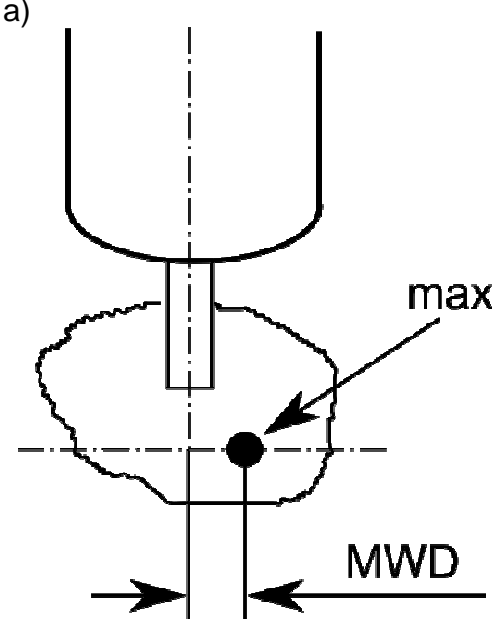

b)

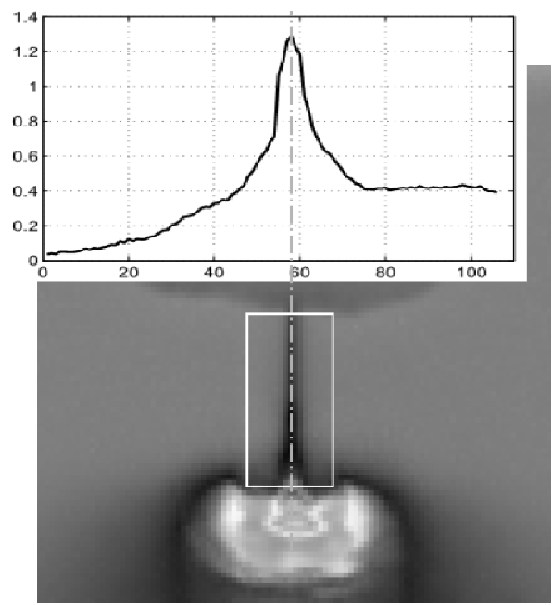

Fig. 9. Idea of determination of horizontal distance between pixel with maximal value and a wire axis of symmetry (a), and way of determination of wire axis of symmetry (b)

\section{Example of application of analysis result of fused images to detection and identification of welding faults}

Image processing and analysis methods presented in the paper were applied to a sequence of images recorded during the welding experiments. Infrared and vision images were analyzed after fusion operation as well as independent, what allowed the comparison of the efficiency of application of image fusion to identification of welding faults. Extracted image features, ordered in time, were used to determine the diagnostic signals which were applied for detection and identification of the welding process instabilities.

State S1
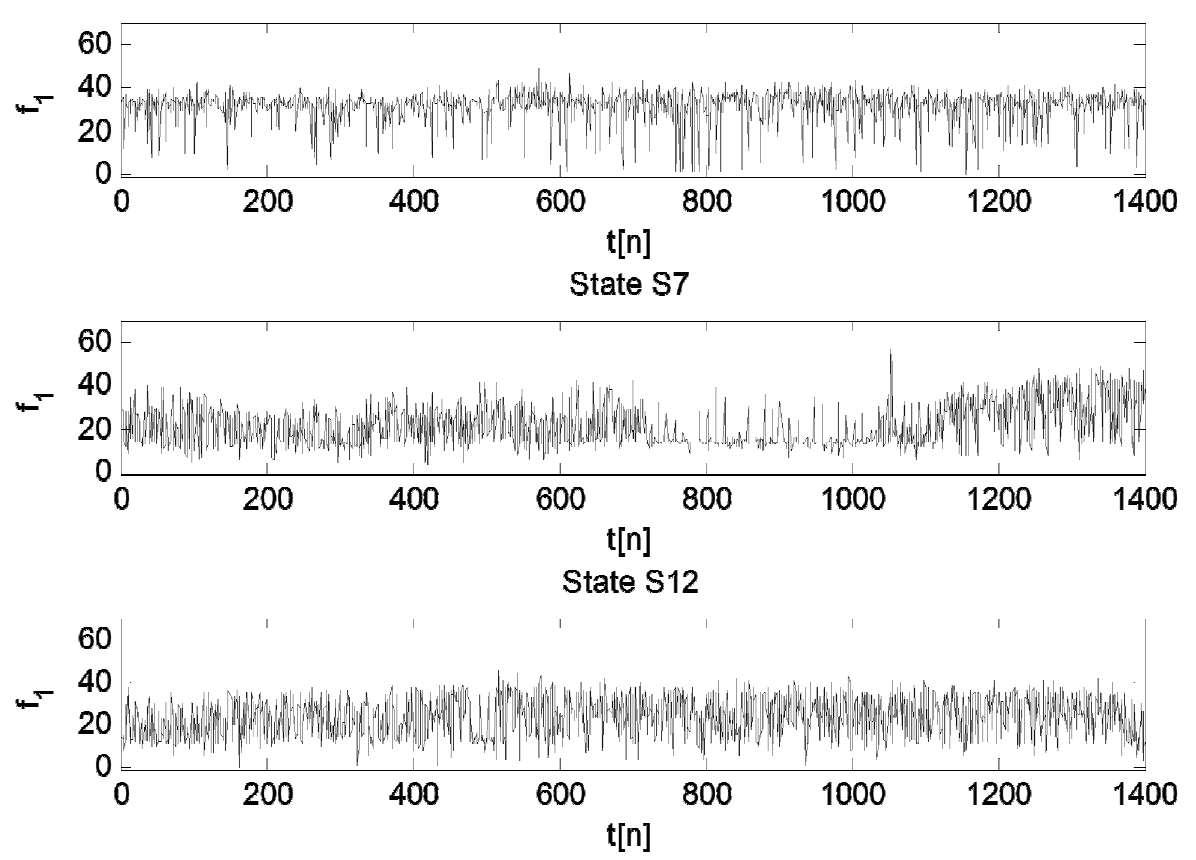

Fig. 10. Waveform of feature values $f 1$ calculated for fused images, representing different welding process instabilities

Exemplary waveforms of feature values representing the width of the central glowing arc $\left(C W=f_{1}\right)$ of fused images are shown in Fig 10. There were compared: the signal representing correct welding process (S1) and the signals corresponding to the welding process in the state S7 (current deviations) and S12 (worn rollers in the wire feeder). It can be noticed, that for the welding process in the state S7 the change of the current is clearly visible in the form of a determined diagnostic signal. However, for the process in state S12, the visual evaluation of diagnostic signals does not allow the unambiguous detection of instability of the welding process. Similar examples occurred during review of diagnostic signals acquired for another states and image features not presented in the article. 
State S9

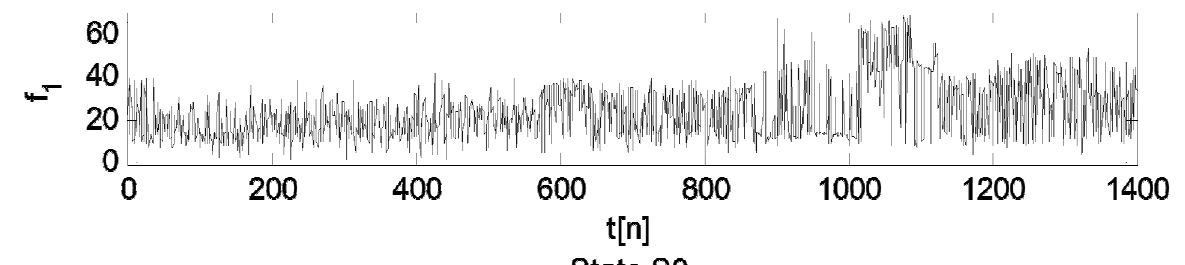

State S9

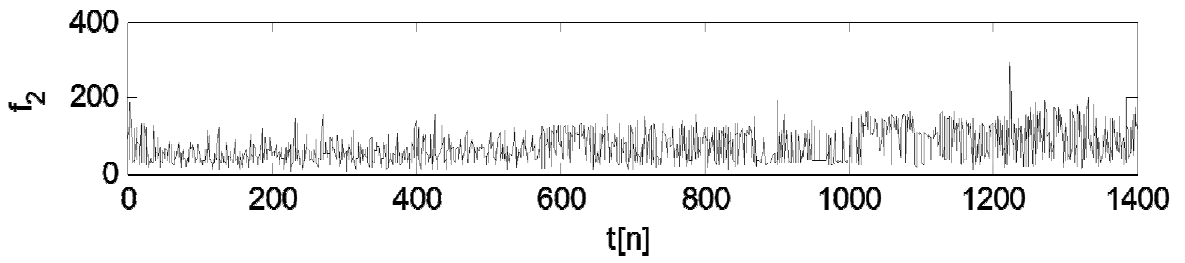

State S9

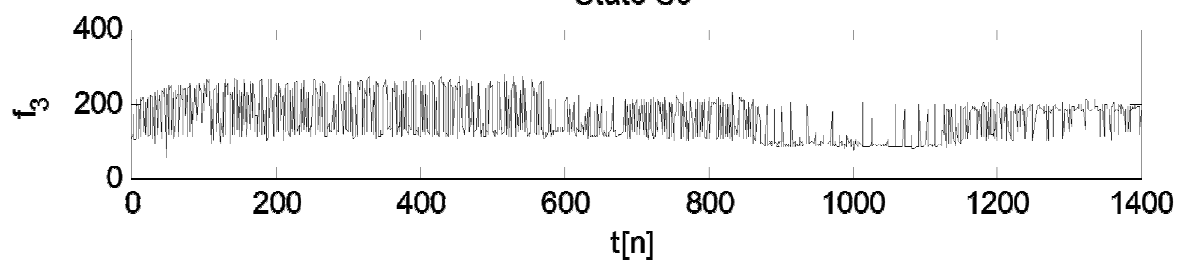

Fig. 11. Waveforms of features $f_{1}, f_{2}, i f_{3}$ extracted from image sequence representing state $S 9$.

Each of the proposed methods of image analysis allowed determining of several sets of features. During the research, feature selection has been made and from each set, features allowing detection and identification of the welding process instabilities were chosen. In the set of statistical features, root mean square (RMS) ( $\left.f_{2}\right)$ proved to be the best feature, in the set of topological features best results were obtained on the basis of characteristics that describes the perimeter $\left(f_{3}\right)$ of the welding arc area. In the case of profile analysis the best feature was the middle width $(C W)\left(f_{1}\right)$. Plots of feature values estimated for the images recorded during the welding process in state S9 (voltage deviations) are shown in Fig. 11. Each of the presented signals allows the detection of simulated instabilities of the welding process. It should be also mentioned, that detected instabilities of the welding process, basing on the analysis of the fused images were not reflected in the quality of welded joint, assessed visually.

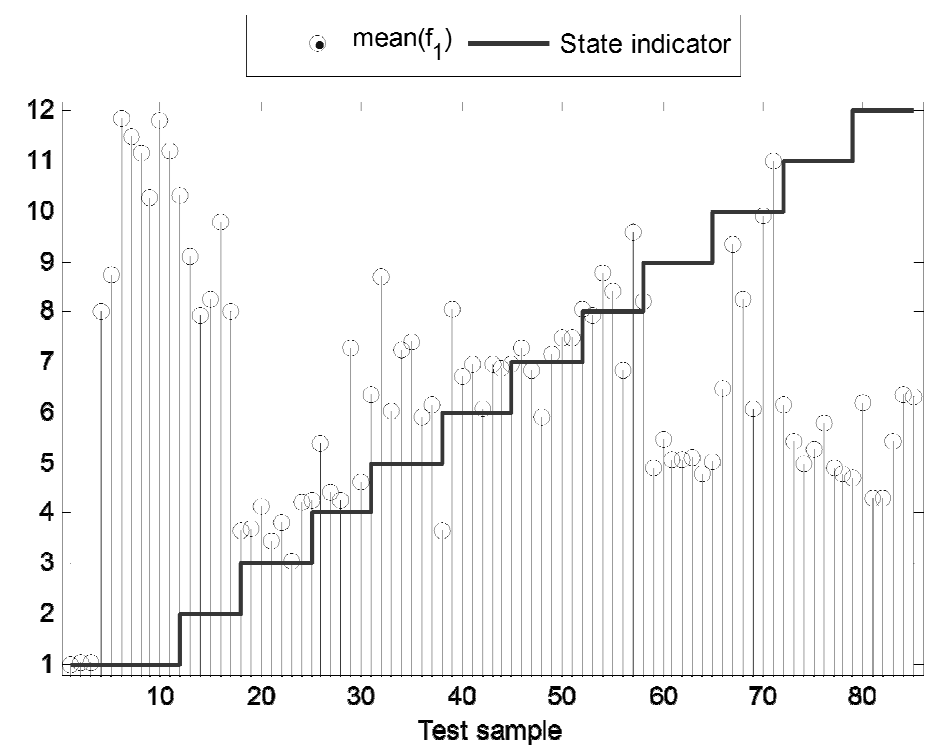

Fig. 12. Exemplary feature values calculated for diagnostics signals representing all simulated process states

Diagnostic signals were assessed using simple statistical estimators such as mean value, RMS, standard deviation, etc. In Fig. 12, the mean values of diagnostic signals obtained on the basis of feature $\left(f_{1}\right)$ of all fused images representing different welding process states are presented. I can be stated that on the basis of the mean values of the diagnostic signals not all classes of simulated welding process can be distinguished. 
Feature values space of the diagnostic signals was used to identification the state of the welding process using the classification process. The classification was carried out using a $k$-nearest neighbours classifier, where the number of neighbours was assumed to $k=7$. Estimation of the efficiency of the classification was performed by leave-one-out classifier error estimation technique. In this method the whole available feature set, containing $\mathrm{N}$ elements was divided into two separate subsets. The training subset contained $N-1$ elements, whereas in the test subset only one element was included. The process of learning and testing of the classifier was performed $\mathrm{N}$ times, so that each of the examples can be found in the test set. A classification accuracy measure $e_{s e n}$ was the relative number of misclassifications calculated using the following formula:

$$
e_{\text {sen }}=\frac{N_{\text {tin }}}{N_{\text {in }}}
$$

where:

$N_{\text {tin }}$ - is the number of correctly classified test examples representing a particular class of state,

$N_{\text {in }}$ - total number of test examples considered and representing a particular class of state.

Classification results obtained for the diagnostic signal obtained by analysis of images after fusion and the independent analysis of thermal images and video images are shown in Fig 13. One can see that classification for twodimensional feature space, of diagnostic signals obtained by analysis of infrared and vision images was also performed.

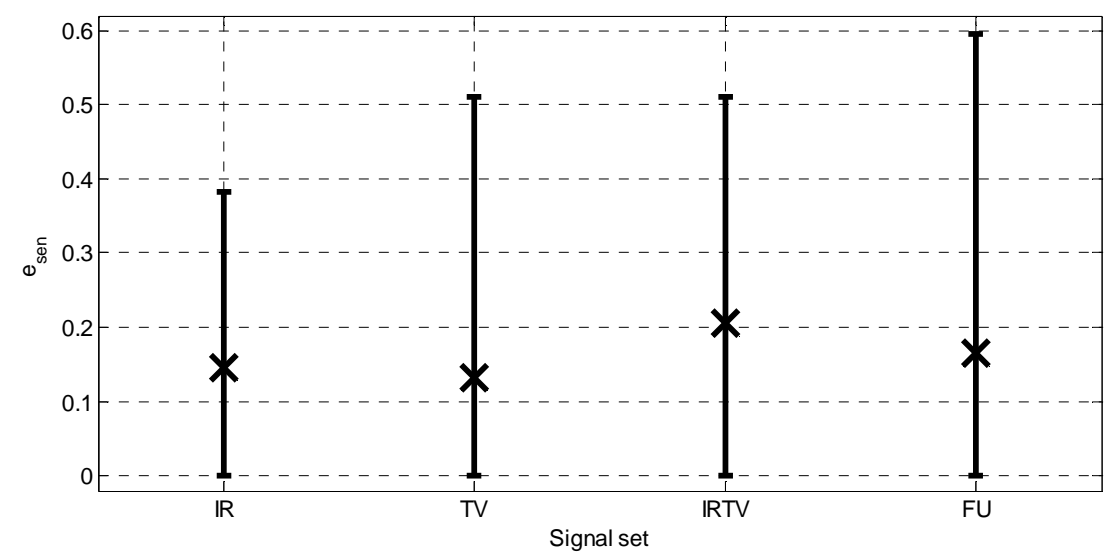

Fig. 13. Mean, minimal, and maximal classification efficiency for features of diagnostics signals obtained from all considered image sequences

Analyzing the results of classification one can see that the best classification results were obtained for feature of diagnostic signals determined by analysis of fused images. The highest efficiency of classification $e_{\text {sen }}=0.59$ was obtained for the mean value of waveform of $f_{1}$ feature, whereas the maximum classification efficiency for the vision images was $e_{\text {sen }}=0.51$ and for the infrared images $e_{\text {sen }}=0.39$. In the case of two-dimensional feature space determined on the basis of infrared and vision images separately, the maximum classification efficiency was $e_{\text {sen }}=0.51$. It can be stated that even by use of such a simple methods of diagnostic signals estimation, the results obtained on the basis of fused images sequences are the best.

\section{Conclusions}

In this paper, several image processing and analysis methods were applied to sequence of fused infrared and vision images of welding arc recorded during different welding experiments. As result of the fused images analysis obtained diagnostic signals, which allowed the detection some class of GMA welding instabilities. Features being the result of the diagnostic signals estimation were applied to identification of welding faults. Investigation of diagnostic signals showed that not all features allows the unambiguous detection of instabilities of the welding process. In this case it is necessary to perform operation of feature selection. Classification results showed also, that processing and analysis of fused images allow the identification of faults of welding process in the satisfying manner. The maximum value of classification efficiency amounted 0,59 was higher from the efficiency of classifications obtained for features of diagnostic signals which were generated as result of the independent analysis of the sequence of infrared and vision images. In authors opinion it is possible to increase of classification efficiency, across optimization of the image segmentation process. Another way of increasing of classification efficiency is to find relevant features of diagnostic signals. Methods of analysis of fused images of welding arc presented in the paper are not optimal and in authors opinion further study on this area and tests of other methods of image processing and analysis are necessary. 


\section{REFERENCES}

[1] Burt P., Kolczynski P., "Enhanced image capture through fusion”, in Proc. 4th Int. Conf. Computer Vision, pp. 173-182, 1993.

[2] Bzymek A., Fidali M., Jamrozik W., Timofiejczuk A.: "Diagnostic vision system for welded joint and welding process assessment”, Problemy Eksploatacji 4/2008, p. 39-52

[3] Dupuis O., Kaftandjian V., Yue Min Zhu, Babot D., "Détection de défauts par fusion de signaux ultrasonores et d'images radiographiques", $17^{\circ}$ colloque GRETSI sur le Traitement des signaux et des images, Vannes, 13-17 septembre 1999.

[4] Fluke White Paper, "Development Insider How patent-pending technology blends thermal and visible light". http://support.fluke.com/find-sales/Download/Asset/2788400 6251 ENG A W.PDF. 20-04-2011

[5] Gonzales R., Wintz P.: "Digital Image Processing". Addison-Wesley Publishing Company, 1987.

[6] Haralick R., Shanmugam K., Dinstein I., "Textural features for image classification", IEEE Transaction on Systems Man Cybernetics, 3(6), 1973, p. 610-621.

[7] Jamrozik W. "An example of surface fault estimation on the basis of super-resolution approach", Diagnostyka, 2010, nr 2(54), pp. 59-64,

[8] Jamrozik W., Fidali M., "Registration methods for infrared and vision images representing dynamically changing scene", Measurement Automation and Monitoring, vol. 57, no. 10/2011, pp. 1134 -1137, (in polish).

[9] Jamrozik W., Fidali M., "Evaluation of the suitability of IR and TV image aggregation algorithms for the purposes of welding process assessment", Proceedings of $11^{\text {th }}$ Quantitative InfraRed Thermography, 11-14 June 2012, Naples, Italy.

[10] Jamrozik W., Fidali M.: "Estimation of image fusion methods for purposes of vision monitoring of industrial process". Measurement Automation and Monitoring, vol. 57, no 09/2011, pp. 993 - 996

[11] Kim Y. S. and Lee J. H. and Ra J. B., 2008, "Multi-sensor image registration based on intensity and edge orientation information", Pattern Recognition, vol. 41, pp. 3356-3365

[12] Kronberger Jr., L., Nicoletti, R., Ranner, G., Graif, E., Stollberger, R., Einspieler, R., Wiltgen, M., Fueger, G.F., Ebner, F. and Steindorfer, P., "Computed fusion of MRI and anti-CEA immunoscintigraphy in the follow up of operated rectal cancer". European Journal of Cancer. v29 iSuppl. 6. S97

[13] Mitianoudis N. and Stathaki T., "Pixel-based and region-based image fusion schemes using ICA bases", Infor. Fusion 8 (2), pp. 131-142, 2007.

[14] Nandhitha N. M., Manoharan N., Rani B. S., Venkataraman B., Sundaram P. K., Raj B.: "Automatic Detection and Quantification of Incomplete Penetration in TIG Welding Through Segmentation and Morphological Image Processing of Thermographs". In: Proc. National Seminar on Non-Destructive Evaluation Dec. 7 - 9, 2006, Hyderabad. 2006. p. 17-22.

[15] Nixon S. M., Aguado S.: "Feature Extraction and Image Processing". Newnes, 2002.

[16] Petrou M., Sevilla P., "Image Processing: Dealing with Texture", Wiley and Sons Inc., 2006.

[17] Renier E., Suzeau P., Truchetet F., Geveaux P.: "Spatial and thermal measurements using Thermography CCD during HF Soldering of metallic tubes". In: Industrial Electronics Society, 1998. IECON '98. Proceedings of the 24th Annual Conference of the IEEE. 1998. p. 1699-1702.

[18] Rockinger, O., "Image Sequence Fusion Using a Shift Invariant Wavelet Transform". Proceedings of the International Conference on Image Processing, vol.3, pp. 288-291, 1997.

[19] Stathaki T., "Image fusion: algorithms and applications", Academic Press, 2008.

[20] Sadjadi F., "Comparative Image Fusion Analysis", Proc. of the 2005 IEEE Computer Society Conference on Computer Vision and Pattern Recognition (CVPR'05) - Workshops, Vol. 03, p.8, 2005.

[21] Venkatraman B., Menaka M., Vasudevan M., Raj B.: "Thermography for Online Detection of Incomplete Penetration and Penetration Depth Estimation", in: 12th Asia-Pacific Conference on NDT, 5th - 10th Nov 2006, Auckland, New Zealand, 2006: pp. 8-12. 